\title{
Endobronchial Sarcoma: A Case Report and Review of Literature
}

\author{
Anu Menon, $M D^{1}$, Varun Shah, $M D^{1^{*}}$, Adnan Hasanovic, $M D^{2}$, Viera Lakticova, $M D^{3}$ \\ ${ }^{1}$ Hofstra Northwell Lenox Hill Hospital, New York, USA \\ ${ }^{2}$ Department of Pathology, Lenox Hill Hospital, New York, USA \\ ${ }^{3}$ Department of Pulmonary, Critical Care, and Sleep Medicine, Northwell Health, New Hyde Park, NY, USA
}

\begin{abstract}
Pulmonary sarcomatoid carcinomas (PSCS) are poorly differentiated non-small cell lung carcinomas (NSCLCs) that, loosely, consist of a sarcoma-like element and/or a sarcomatous component - they represent one of the rarest sub-types of lung tumors. Pre-operative analysis with bronchoscopic biopsy is often an underrepresentation of tissue structure in its entirety, leading to hindrance in definitive diagnosis. Here we present the case of a male presenting with progressive dyspnea, ultimately diagnosed with a pulmonary sarcomatoid tumor with extensive local infiltration on bronchoscopy. Given the relatively low incidence of this cancer, we discuss the epidemiology, risk factors, and diagnostic tools used in characterization of tumors. Most pulmonary sarcomatoid carcinomas contain features and immunophenotypes consistent with conventional NSCLC, lending itself to the idea that this group of tumors falls along a spectrum that represents common origin from a totipotent stem cell. This allows for alignment of a cancer with typically poor prognosis and limited treatment options with one with greater possibilities for therapeutic intervention. Despite the ultimate decision for palliation seen in this case, it is of value for clinicians to be aware of the focus on research into the areas of molecular targeted therapy and immunotherapy for treatment of pulmonary sarcomatoid carcinomas, given success with treatment of non-small cell lung cancers.
\end{abstract}

\section{Introduction}

Pulmonary sarcomatoid carcinomas (PSCs) are poorly differentiated non-small cell lung carcinomas (NSCLCs) that, loosely, consist of a sarcoma-like element and/or a sarcomatous component - they represent one of the rarest sub-types of lung tumors [1]. Pre-operative analysis with bronchoscopic biopsy is often an underrepresentation of tissue structure in its entirety, leading to hindrance in definitive diagnosis [2]. Here we present the case of a male presenting with progressive dyspnea, ultimately diagnosed with a pulmonary sarcomatoid tumor with extensive local infiltration on bronchoscopy. Given the relatively low incidence of this cancer, we discuss the epidemiology, risk factors, and diagnostic tools used in characterization of tumors. Most pulmonary sarcomatoid carcinomas contain features and immune phenotypes consistent with conventional NSCLC, lending itself to the idea that this group of tumors falls along a spectrum that represents common origin from a totipotent stem cell [3]. This allows for alignment of a cancer with typically poor prognosis and limited treatment options with one with greater possibilities for therapeutic intervention [4]. Despite the ultimate decision for palliation seen in this case, it is of value for clinicians to be aware of the focus on research into the areas of molecular targeted therapy and immunotherapy for treatment of pulmonary sarcomatoid carcinomas, given success with treat- ment of non-small cell lung cancers [4].

\section{Case}

A 71-year-old male, with a 100 pack year smoking history, presented to an outside hospital with 2-weeks of progressive dyspnea. Additional past medical history was notable for COPD, hypertension, diabetes mellitus, bladder cancer status post resection, and chronic kidney disease. Social history was negative for alcohol use, he quit smoking 4 years ago, and was a retired accountant. At the time of his evaluation, he denied chest pain, hemoptysis, fevers and chills. Vital signs were significant for hypoxia to $86 \%$ on room air, along with tachypnea and tachycardia. Patient was noted to have absent breath sounds in the entirety of the left lung field with some mild expiratory wheezing in the right lung field. Basic

*Corresponding author: Varun Shah, MD, Hofstra Northwell Lenox Hill Hospital, 100 East 77th Street, NY, 10075, USA

Accepted: October 19, 2020

Published online: October 21, 2020

Citation: Menon A, Shah V, Hasanovic A, et al. (2020) Endobronchial Sarcoma: A Case Report and Review of Literature. Ann Lung Cancer 3(1):90-93 
Citation: Menon A, Shah V, Hasanovic A, et al. (2020) Endobronchial Sarcoma: A Case Report and Review of Literature. Ann Lung Cancer 3(1):90-93

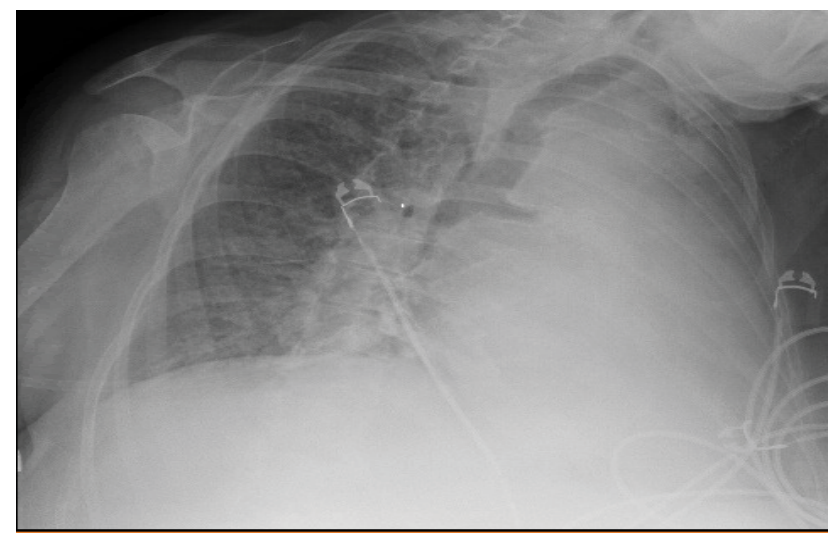

Figure 1: Portable chest radiograph showing complete opacification of the left hemithorax.

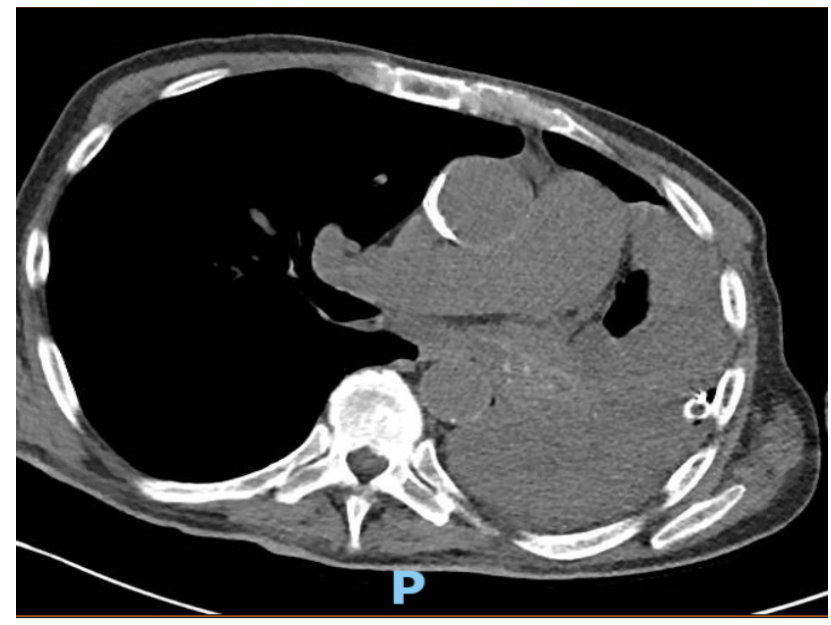

Figure 2: $\mathrm{CT}$ chest with mediastinal window showing complete obstruction of the left mainstem bronchus.

Note: Some calcification (white arrow) noted within the tumor in the bronchus.

blood work, including differential cell count and comprehensive metabolic panel, was normal except for the patient's known chronic renal dysfunction. Chest radiograph (Figure 1) was notable for a large left pleural effusion. A chest tube was subsequently placed, draining $1.5 \mathrm{~L}$ in totality, which provided symptomatic relief. Pleural fluid studies were negative for malignancy and fluid was consistent with a transudate based on Light's criteria. Non-contrast chest CT scan (Figure 2) revealed a left main stem bronchus endobronchial lesion and a small residual pneumothorax. The patient then underwent bronchoscopy with endobronchial biopsy, revealing inflammatory squamous epithelial cells, but no malignant cells. The patient was subsequently transferred to our facility where he underwent rigid bronchoscopy. The left main stem bronchus exhibited complete obstruction by an adherent mass (Figure 3), coated in thick secretions proximally. Tumor de-bulking was attempted with grasper forceps using hot diathermy. The tumor was noted to have a gritty consistency and was seemingly resistant to de-bulking. De-bulking was attempted once more with Nd: YAG laser along with Fogarty balloon dilation,

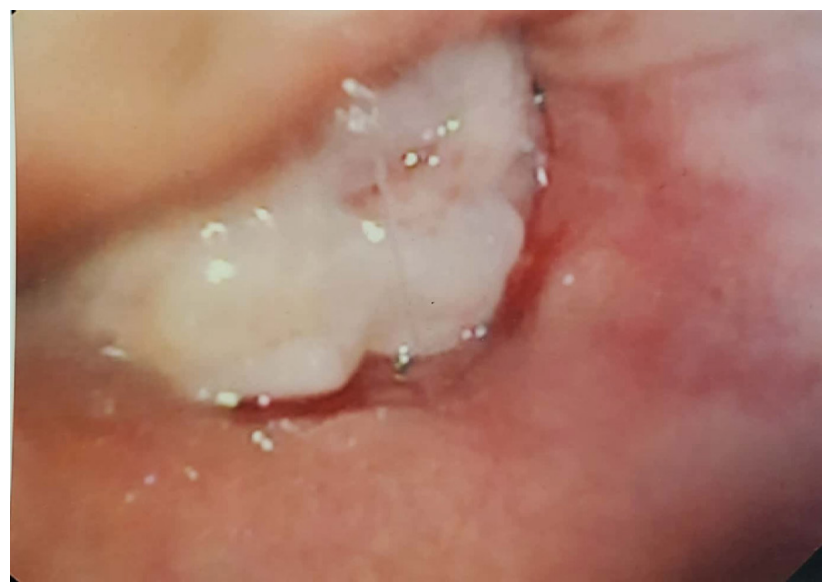

Figure 3: Rigid bronchoscopy image showing the left mainstem bronchus with complete obstruction by an irregularly shaped, whitish colored endobronchial mass with a gritty consistency.

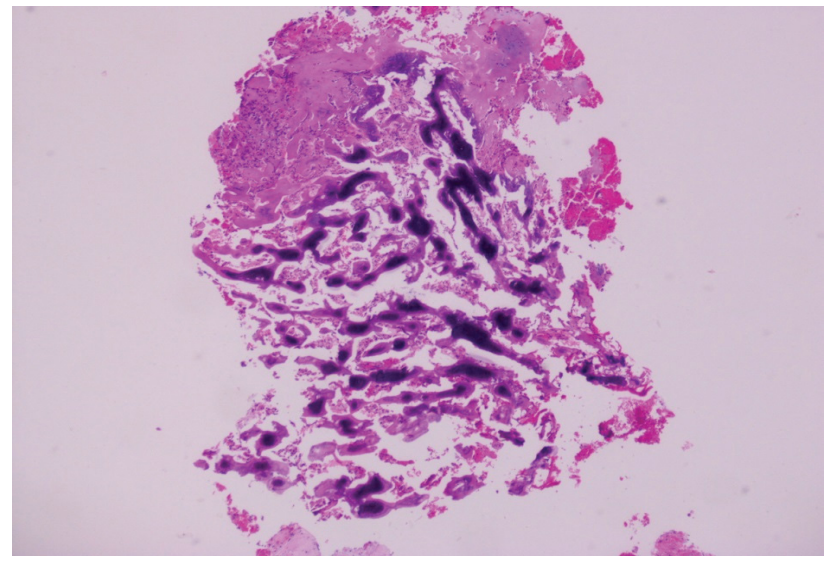

Figure 4: Hematoxylin and eosin stain under low magnification (25x) showing a proliferation of atypical spindle cells in a background of immature bone.

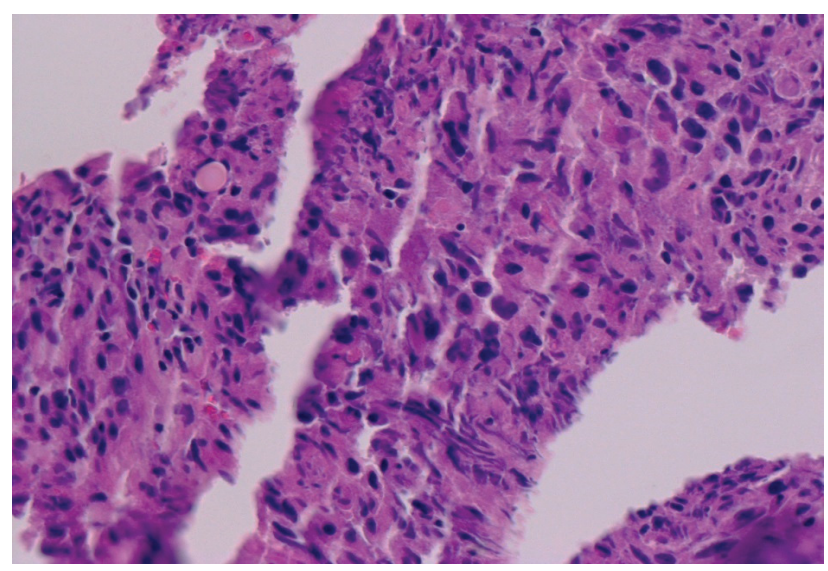

Figure 5: Hematoxylin and eosin in medium power view (40x) of the endobronchial biopsy showing bone formation with intermixed spindle cells.

resulting in merely minimal recanalization. Unfortunately, given the extent of the tumor, proximity to the pulmonary 
artery, adherence to the bronchial wall, and tumor consistency complete recanalization was impossible. Surgical pathology showed atypical spindle cell proliferation with immature bone formation that was intermixed. Immunohistochemical staining of the spindle cell component revealed special ATrich sequence-binding protein 2 (SATB2), p40 and S 100 (Figure 4, Figure 5 and Figure 6).

\section{Discussion}

Pulmonary sarcomatoid carcinomas (PSCS) are poorly differentiated non-small cell lung carcinomas (NSCLCs) that, loosely, consist of a sarcoma-like element (malignant spindle or giant cells) and/or a sarcomatous component (bone, cartilage, or muscle) [1]. They serve as one of the rarest sub-types of lung tumors, accounting for only $0.1-0.4 \%$ of all pulmonary malignancies [2]. Given the relatively low incidence and histopathologic heterogeneity described, identification is often cumbersome and fraught with difficulty. This is further compounded by the fact that pre-operative analysis with bronchoscopic biopsy is often an underrepresentation of tissue structure in its entirety, leading to hindrance in definitive diagnosis. Disease is more common in males, particularly those who identify as active or past smokers with a moderate to heavy history of tobacco use. Peak age at presentation tends to be within the sixth and seventh decade of life. The above characteristics correspond exactly to the profile of our patient.

Grossly, tumors present as either central or peripheral lesions, commonly in the right upper lobe. Growth is brisk, often with local invasion of the bronchial tree, pulmonary parenchyma and adjacent anatomical structures [2]. Therefore, subsequent symptomatology is typically a manifestation of this degree of encroachment. In general, presenting symptoms are similar to those of NSCLC and include: cough, dyspnea, chest pain, and hemoptysis [5]. Incidentally found tumors, with patients remaining asymptomatic, are rare and represent only $21 \%$ of cases [6]. The differential diagnoses of pulmonary sarcomatoid carcinomas must include any primary or secondary high-grade tumor of the lung, including conventional NSCLC. Specific consideration must be given to true sarcomas of the lung, a relatively rare diagnosis, and sarcoma-

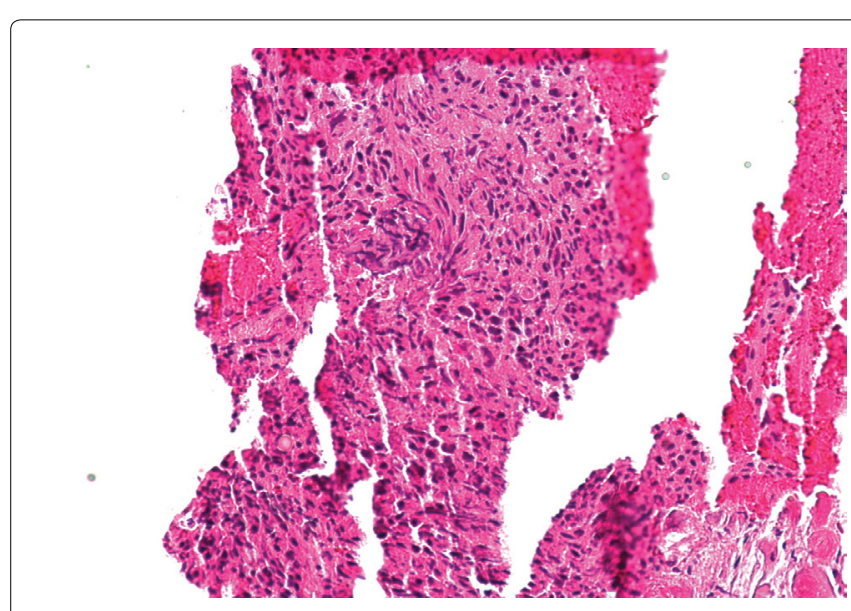

Figure 6: Hematoxylin and eosin stain under medium magnification (40x) showing a cluster of markedly atypical spindle cells. toid mesothelioma with significant pleural involvement. Pure sarcomas are mesenchymal in origin and lack an epithelial component; therefore, if reactive markers for mesenchymal rather than epithelial differentiation are present, a diagnosis of sarcoma should be pursued [7]. The above harbor disappointing prognoses, prompting the question of whether it is even fruitful to differentiate a diagnosis of pulmonary sarcomatoid carcinoma - however, it is universally understood that continued efforts at honing identification and classification will ultimately yield greater success in treatment strategy [6].

The most common appearance on imaging of PSCs is a large, peripheral upper lobe mass with signs of local invasion [2]. Contrast enhanced computed tomography (CT) scans typify large, heterogeneous lesions with the appearance of dense, soft tissue rife with patchy necrosis. Irregular thickwalled cavities may develop given uneven distribution of necrotic tissue. Rare cases have been described of presentation as single, necrotizing cavitary lesions on CT Scan, masquerading as presumptive infectious or autoimmune phenomenon [8]. Bullous lesions with indwelling soft tissue masses, mistaken for as per gilloma, but ultimately revealing of sarcomatoid carcinoma, have also been reported. Differentiation lies in presence of a stalk attaching the mass to the wall of the cavity, distinguishing tumor from a mobile as per gilloma [9]. Calcification within the tumor is an infrequent finding [10]. Using fluorine-18 fluorodeoxy glucose PET/CT, sarcomatoid carcinomas typically present with an intense uptake (SUV max of about 15). In distinguishing pulmonary sarcomatoid carcinomas from other differentials, chest imaging is useful in that it potentially elucidates mesothelioma, which typically presents with diffuse pleural involvement rather than as a solitary parenchymal mass [11]. Furthermore, imaging can expose the presence of a primary tumor that is extra-pulmonary with metastatic involvement, which is most often the case with pure sarcomas [12].

Historically, diagnosis has been made with methodical histopathologic analysis via light microscopy. Increasingly, however, for the management of lung cancers as a whole, immune histochemistry has become the standard of care given its role in guiding therapy [1]. The observation that most pulmonary sarcomatoid carcinomas (aside from carcinosarcoma and pulmonary blastoma) contain features and immune phenotypes consistent with conventional NSCLC lends itself to the idea that this group of tumors falls along a spectrum that represents common origin from a totipotent stem cell with subsequent epithelial-mesenchymal differentiation [3]. Application of modern immune histochemical techniques allows for alignment of sarcomatoid carcinomas with the more conventional NSCLCs, for which treatment is more readily delineated, and early identification of cases that would benefit from molecular testing and targeted therapy.

Surgery in early, localized disease provides the greatest overall survival benefit and remains the standard of care in this select patient population. However, the role of resection remains questionable given the disease's high recurrence rate [13]. The efficacy of systemic chemotherapy, typically platinum based regimens akin to those used for NSCLC, is varied, with some studies illustrating modest benefit while 
others reveal no improvement in survival [14]. No significant role has been shown for chemotherapy in advanced disease [13]. Molecular targeted therapies and immunotherapy are increasingly being studied given the role they have garnered in management of NSLC. PSCs are clonal tumors and around $70 \%$ of cases may present with demonstrable mutations (ex: EGFR mutation, ALK translocation, MET amplification), with nearly $40 \%$ expressing 4 or more mutations. $70-90 \%$ of tumors display PD-L1 expression - immune checkpoint inhibitors targeting this protein have proven efficacious in NSLC treatment, however, this has yet to be validated in trials studying PSCs [4]. With alignment of PSCs along the spectrum of NSLC and comparison of specific gene expression patterns in these two groups, molecular analysis of immune markers and mutations broadens the array of treatment possibilities in severe disease.

\section{Conclusion}

A rare sub-type of lung cancer which carries significantly high mortality, PSCs are poorly differentiated NSCLCs that comprise of both a sarcoma-like element and a sarcomatous element. Chest imaging can help delineate the extent of the tumor and local invasion, while a PET scan can assist in identifying distal metastases, if any. Unfortunately, definitive diagnosis is hindered by need for tissue analysis via surgical resection as bronchoscopic biopsy generally leads to inadequate sampling. The onus of diagnosis ultimately falls on histopathologic examination and recent advances in immune histochemistry, which has led to the emergence of new classification systems. These serve to align the often elusive PSCs with the more readily diagnosed NSCLCs in an attempt to strategize potentially successful treatment regimens via further molecular testing. This arena of research requires further investigation as the prognosis for PSCs at present remains poor. In the patient that we presented, PET Scan was performed for staging and did not uncover extra-pulmonary spread. Ultimately, due to the extensive nature of malignancy and failure to wean from the ventilator, the patient opted for palliation and expired shortly thereafter.

\section{Conflicts of Interest}

Authors have no conflicts of interest to disclose.

\section{References}

1. Travis WD, E Brambilla, AG Nicholson, et al. (2015) The 2015 World Health Organization Classification of Lung Tumors: Impact of Genetic, Clinical and Radiologic Advances Since the 2004 Classification. J Thorac Oncol 10: 1243-1260.

2. Travis WD (2010) Sarcomatoid neoplasms of the lung and pleura. Arch Pathol Lab Med 134: 1645-1658.

3. Weissferdt A (2018) Pulmonary Sarcomatoid Carcinomas: A Review. Adv Anat Pathol 25: 304-313.

4. Kotlowska MP, AG Rueda, ME Olmedo, et al. (2019) Efficacy of immunotherapy in sarcomatoid lung cancer, a case report and literature review. Respir Med Case Rep 26: 310-314.

5. Arshad HS, RA Dudekula, M Niazi, et al. (2017) A Rare Case of Sarcomatoid Carcinoma of the Lung with Spine Metastasis, Including a Literature Review. Am J Case Rep 18: 760-765.

6. Pelosi G, A Sonzogni, T De Pas, et al. (2010) Review article: pulmonary sarcomatoid carcinomas: A practical overview. Int J Surg Pathol 18: 103-120.

7. Franks TJ, JR Galvin (2010) Sarcomatoid carcinoma of the lung: histologic criteria and common lesions in the differential diagnosis. Arch Pathol Lab Med 134: 49-54.

8. Gaurang Nandkishor Vaidya, Rushikesh Shah, Amit Dhamoon, (2017) Pulmonary sarcomatoid carcinoma presenting as a necrotizing cavitary lung lesion: diagnostic dilemma. The Journal of Community and Supportive Oncology 15: 103-105.

9. Aggarwal Nidhi, Paul Vishesh, Chawla Kabu, et al. (2014) Sarcomatoid Carcinoma of Lung Masquerading as Aspergilloma. Chest 146.

10. Qin Z, B. Huang, G. Yu, et al. (2019) Gingival metastasis of a mediastinal pulmonary sarcomatoid carcinoma: a case report. J Cardiothorac Surg 14: 161.

11. Sureka B, B.B. Thukral, MK Mittal, et al. (2013) Radiological review of pleural tumors. Indian J Radiol Imaging 23: 313-320.

12. Billingsley K.G., M.E. Burt, E. Jara, et al. (1999) Pulmonary metastases from soft tissue sarcoma: analysis of patterns of diseases and postmetastasis survival. Ann Surg 229: 602-610.

13. Karim N.A., J. Schuster, I. Eldessouki, et al. (2018) Pulmonary sarcomatoid carcinoma: University of Cincinnati experience. Oncotarget 9: 4102-4108.

14. Ouziane I., S. Boutayeb, H. Mrabti, et al. (2014) Sarcomatoid carcinoma of the lung: a model of resistance of chemotherapy. $\mathrm{N}$ Am J Med Sci 6: 342-345.

DOI: $10.36959 / 825 / 584$

Copyright: (C) 2020 Menon A, et al. This is an open-access article distributed under the terms of the Creative Commons Attribution License, which permits unrestricted use, distribution, and reproduction in any medium, provided the original author and source are credited. 